\title{
FRECUENCIA DE LOS ANTÍGENOS DEL SISTEMA Rh EN DONANTES DE SANGRE Rh D NEGATIVO
}

\section{FREQUENCY OF THE ANTIGENS OF THE SYSTEM Rh IN BLOOD DONORS Rh D NEGATIVE}

\author{
Sandra Milena Zuluaga $\mathrm{G}^{1}$, Yesica María Gándara $\mathrm{H}^{2}$, Rossana Villegas $\mathrm{G}^{3}$ \\ Recibido para publicación: Mayo 17 de 2017 - Aprobado para publicación: Junio 28 de 2017
}

\begin{abstract}
RESUMEN
Objetivo: Determinar la frecuencia de los antígenos del sistema $\mathrm{Rh}$ en donantes de sangre Rh $\mathrm{D}$ negativo. Materiales y métodos: Estudio retrospectivo y descriptivo donde se revisaron la totalidad de registros de donantes Rh D negativo del Banco de Sangre de Córdoba entre los años 2012-2015. Para describir las características sociodemográficas de los donantes Rh D negativo e identificar la frecuencia de los fenotipos del sistema Rh se calcularon frecuencias absolutas, relativas e intervalos de confianza para las proporciones del 95\%, para comparar los fenotipos del sistema Rh de acuerdo al grupo ABO y las variables sociodemográficas se calcularon frecuencias absolutas, relativas, intervalos de confianza del $95 \%$ y la prueba Chi cuadrado En los análisis se consideraron significativos valores $p<0,05$ y la información se almacenó y analizó en SPSS versión 23. Resultados: EI fenotipo hallado con mayor frecuencia fue ccee, que correspondió a un $92 \%$, los otros fenotipos se encontraron en porcentajes más bajos distribuidos de la siguiente forma: Ccee 5,9\%, ccEe 1,2\%, CCee 0,3\%, y CcEe 0,2\%. El 88\% de las donaciones fueron realizadas por donantes del género masculino y se encontró que el grupo sanguíneo $\mathrm{ABO}$ con mayor frecuencia fue el $\mathrm{O}$ correspondiente a un $66,4 \%$. Conclusión: La frecuencia a nivel departamental coincide con los resultados encontrados a nivel nacional e internacional.
\end{abstract}

Palabras clave: Sistema de grupo sanguíneo $\mathrm{Rh}-\mathrm{Hr}$, fenotipo, sistema del grupo sanguíneo $A B O$, complejo antígeno - anticuerpo.

\begin{abstract}
Objective: To determine the frequency of antigens of the $\mathrm{Rh}$ system in donors of Rh $\mathrm{D}$ negative blood. Materials and methods: Retrospective and descriptive study where all the records of $\mathrm{Rh} D$ negative blood donors from the Blood Bank of Cordoba between the years 2012-2015 were reviewed. To describe the sociodemographic characteristics of $\mathrm{Rh}$ D negative blood donors and to identify the frequency of phenotypes of the Rh system, absolute, relative and confidence intervals were calculated for $95 \%$ ratios to compare Rh system phenotypes according to the $A B O$ group And sociodemographic variables, absolute, relative frequencies, $95 \%$ confidence intervals and Chi square test were calculated. $p$ values $<0.05$ were considered significant and the information was stored and analyzed in SPSS version 23. Results: The phenotype found most frequently was ccee, which corresponded to $92 \%$; the other phenotypes were found in lower percentages distributed as follows: Ccee $5.9 \%$, ccEe $1.2 \%$, CCee $0.3 \%$, and CCEE $0.2 \% .88 \%$ of the donations were made by male donors and the $A B O$ blood group with the highest frequency was found to be $O$ corresponding to $66.4 \%$. Conclusion: Both nationally and globally, the most frequent phenotype for $\mathrm{Rh} \mathrm{D}$ negative people is ccee, with a slight variation in their percentage of repetition between regions.
\end{abstract}

Keywords: Rh Blood Group System, phenotype, ABO blood group system, Antigen-antibody complex.

\footnotetext{
${ }^{1}$ Bacterióloga. Universidad de Córdoba

${ }^{2}$ Bsc. Docente programa de Bacteriología, Universidad de Córdoba

${ }^{3}$ M.Sc. Docente programa de Bacteriología, Universidad de Córdoba. Grupo de Investigaciones Microbiológicas y Biomédicas de Córdoba-GIMBIC
} 


\section{INTRODUCCIÓN}

Actualmente, la medicina transfusional es una de las herramientas terapéuticas más implementadas en el tratamiento de distintas patologías que afectan la salud en todas las poblaciones a nivel mundial (1).

Debido al gran polimorfismo, la alta inmunogenicidad y la importancia clínica que tiene el sistema Rh luego del sistema ABO (2), se debe contar con bases estadísticas claras del comportamiento y frecuencia de los antígenos pertenecientes a dicho sistema, relacionando estos con el tipo de población de una región para prevenir reacciones transfusionales que comprometan la vida de los receptores.

El sistema Rh es uno de los más importantes clínicamente por su poder inmunogénico porque produce severas reacciones hemolíticas. Es un sistema con gran polimorfismo, dado que cuenta con 44 o más antígenos, es el más grande de los 30 sistemas de grupos sanguíneos según la Sociedad Internacional de Transfusión de Sangre (3). Después del antígeno $D$, le siguen en capacidad inmunogénica por orden decreciente el c, E, e, y el C (4).

Por lo tanto continua siendo la transfusión de componentes sanguíneos un reto para quienes ejercen la práctica, ocasionada por el riesgo que trae consigo la aplicación de los mismos (1).

En los últimos años se han establecido medidas preventivas en los bancos de sangre para facilitar la disponibilidad en la entrega de hemocomponentes, cada vez se aplican nuevos controles y procedimientos que garantizan la seguridad transfusional (5).

Conforme a lo citado, se hace necesario hacer fenotipificación a todos los donantes Rh D negativos, para de esa manera prevenir posibles isoinmunizaciones en los receptores de glóbulos rojos y reducir el riesgo de reacciones post-tras- nfusionales. El objetivo de este estudio fue determinar la frecuencia de los antígenos del sistema $\mathrm{Rh}$ en donantes de sangre Rh $\mathrm{D}$ negativo.

\section{MATERIALES Y MÉTODOS}

Se realizó un estudio de tipo descriptivo retrospectivo, donde se tomaron los datos del software e-Delphyn, de las personas que durante cuatro años (2012-2015) acudieron como donantes al Banco de Sangre de Córdoba. La población estuvo constituida por todos los donantes efectivos en el período establecido, en total 39842. La muestra fueron todos los donantes Rh D negativo, en total 1686. Se hizo una revisión de los registros para tomar los datos epidemiológicos. La información recolectada fue organizada en tablas en Excel, y se organizó de la siguiente manera: sexo, edad, grupos sanguíneos y antígenos del sistema Rh. Para describir las características sociodemográficas de los donantes $R$ h $D$ negativo e identificar la frecuencia de los fenotipos del sistema Rh se calcularon frecuencias absolutas, relativas e intervalos de confianza para las proporciones del $95 \%$, para comparar los fenotipos del sistema $\mathrm{Rh}$ de acuerdo al grupo $A B O$ y las variables sociodemográficas se calcularon frecuencias absolutas, relativas, intervalos de confianza del $95 \%$ y la prueba Chi cuadrado. Para las variables cuantitativas se calcularon medidas de resumen (tendencia central, posición y dispersión). En los análisis se consideraron significativos valores $p<0,05$ y la información se almacenó y analizó en SPSS versión 23.

De acuerdo con la la Resolución 008430 de Octubre 4 de 1993, en la que se establecen las normas científicas, técnicas y administrativas para la investigación en salud en Colombia, este estudio se clasifico como riesgo mínimo por incluir solo procedimientos con baja probabilidad de afectar a los individuos participantes. 


\section{RESULTADOS}

Se analizaron en total 39842 registros de donantes, de los cuales el 4,2\% (1686) fueron donantes efectivos Rh D Negativo. La edad promedio fue de $34 \pm 10,2$ años, la edad mínima fue 18 y la máxima 65 años (Tabla 1 ).

Tabla 1. Frecuencia de edad en los donantes.

\begin{tabular}{lcc}
\hline Grupo etario & $\begin{array}{c}\text { Frecuencia } \\
\text { absoluta }\end{array}$ & $\begin{array}{c}\text { Frecuencia } \\
\text { relativa }\end{array}$ \\
\hline $\mathbf{1 8 - 2 8}$ & 572 & $33,9 \%$ \\
$\mathbf{2 9 - 3 8}$ & 576 & $34,1 \%$ \\
$\mathbf{3 9 - 4 8}$ & 375 & $22,2 \%$ \\
$\mathbf{4 9 - 5 8}$ & 148 & $8,8 \%$ \\
$\mathbf{5 9 - 6 5}$ & 15 & $0,9 \%$ \\
Total & 1686 & $100 \%$ \\
\hline
\end{tabular}

Al determinar las frecuencias del grupo sanguíneo $A B O$ que presentó la muestra de estudio, se encontró que el más común fue el $O$ con un porcentaje de $67,4 \%$ y en menor proporción el grupo $A B$ con un $0,8 \%$ de las donaciones (Tabla 2).

El fenotipo encontrado con mayor frecuencia fue el ccee con el 92,3\%, seguido de Ccee con $5,9 \%$, otros fenotipos menos frecuentes fueron $c c E e, C C e e, C c E e, c c E E, C c E E$ y $C C E E$. (Tabla 3)

Tabla 2. Frecuencias absoluta y relativa del grupo sanguíneo $A B O$ en las donaciones $R h D$ negativo.

\begin{tabular}{lrc}
\hline Grupo ABO & $\begin{array}{c}\text { Frecuencia } \\
\text { absoluta }\end{array}$ & $\begin{array}{c}\text { Frecuencia } \\
\text { relativa }\end{array}$ \\
\hline O & 1137 & $67,4 \%$ \\
A & 412 & $24,4 \%$ \\
B & 123 & $7,3 \%$ \\
AB & 14 & $0,8 \%$ \\
Total & 1686 & $100 \%$ \\
\hline
\end{tabular}

Tabla 3. Fenotipos del sistema Rh.

\begin{tabular}{lrr}
\hline Fenotipos Rh & $\begin{array}{c}\text { Frecuencia } \\
\text { absoluta }\end{array}$ & $\begin{array}{c}\text { Frecuencia } \\
\text { relativa }\end{array}$ \\
\hline ccee & 1556 & $92,3 \%$ \\
Ccee & 99 & $5,9 \%$ \\
$c c E e$ & 20 & $1,2 \%$ \\
$C C e e$ & 5 & $0,3 \%$ \\
CcEe & 3 & $0,2 \%$ \\
$c c E E$ & 1 & $0,0 \%$ \\
$C c E E$ & 1 & $0,0 \%$ \\
$C C E E$ & 1 & $0,0 \%$ \\
Total & 1686 & $100 \%$ \\
\hline
\end{tabular}

Al comparar los fenotipos Rh más comunes según las características sociodemográficas (género y edad) se encontró predominio del fenotipo ccee, tanto en hombres como en mujeres, seguido del Ccee. Al igual en los diferentes grupos etarios se mantuvo el predominio del fenotipo ccee.

\section{DISCUSIÓN}

La frecuencia de los fenotipos del sistema $\mathrm{Rh}$ para individuos $\mathrm{Rh} \mathrm{D}$ Negativo es poco conocida, tal vez porque los fenotipos diferentes a ccee (los que poseen antígeno $C$ o antígeno $E)$ se encuentran en aproximadamente 1 de cada 1000 donantes, sin embargo, adquieren gran relevancia cuando logran sensibilizar a un paciente (8). Se conocen frecuencias de fenotipos del sistema $\mathrm{Rh}$ a nivel mundial que oscilan entre $90.1 \%$ y el $89.9 \%$ donde el más frecuente es el ccee seguido de Ccee $(6,7,8)$. Resultados similares da a conocer esta investigación, al igual que lo demuestra otra investigación realizada en Arabia Saudita donde la frecuencia de antígenos c y e son altos (74,3 y $95 \%)$, mientras que las frecuencias de $C$ y E son $62,3 \%$ y $23,5 \%$, respectivamente (7). $\mathrm{Al}$ comparar los fenotipos Rh más comunes según las características sociodemográficas (género 
y edad) se encontró predominio del fenotipo ccee, seguido del Ccee.

En relación a la edad existe un estudio de Caracterización clínica y hematológica de donantes a repetición de un banco de sangre de Medellín, donde el $64,2 \%$ de los donantes correspondieron a adultos jóvenes (21-45 años) (9), cifra similar a la de esta investigación donde solamente $56,1 \%$ de los donantes del presente estudio están en el rango de edad entre los 18 a 48 años y existe un bajo porcentaje en la población de adultos medios, lo cual se relaciona con ideas erróneas o mitos de la donación de sangre, por ejemplo, miedo a la transmisión de enfermedades infecciosas o consecuencias físicas indeseadas luego de la flebotomía según datos encontrados en una investigación realizada en Guatemala en los años 2010 y 2011 (6), lo cual también se evidencia en otro estudio realizado en 15 paises dentro de ellos Colombia, por García y colaboradores en el año 2003, donde la mayoría de donadores latinoamericanos poseen conocimientos generales sobre la donación y transfusión de sangre, pero tiene una comprensión relativamente limitada de aspectos específicos sobre el proceso (10).

La investigación encontró un mayor porcentaje de donantes de sexo masculino, dato que coincide con la OMS, en su informe de 2017 donde las mujeres efectúan el 30\% de las donaciones, si bien este porcentaje es muy variable. En 18 de los 118 países que informaron de estas cifras, menos del $10 \%$ de las donaciones se recogieron de mujeres (12). Esto no está relacionado con el hecho de que las mujeres sean menos generosas, sino que tienden a tener menos reservas de hierro que los hombres; además, no pueden donar cuando están embarazadas y son diferidas por 6 meses y hasta 1 año si están lactando. A las donantes del sexo femenino también se les difiere por 24 horas cuando presenta cólico menstrual, y en caso de sangrado profuso se aceptan luego de
24 horas de que éste haya cesado. Todo esto las hace menos elegibles (13).

Con relación a la frecuencia de grupo sanguíneo ABO se observó un comportamiento similar a lo reportado en la literatura, en un trabajo realizado por Cruz Bermudez et al (11), en Tunja-Colombia, mostró que el $62,9 \%$ de los donantes eran del grupo $\mathrm{O}$, el $27,1 \%$ del grupo $A$, el $8,5 \%$ del grupo $B$ y el $1,5 \%$ para el grupo $A B$, corroborando dicha frecuencia de acuerdo a lo establecido en la literatura.

Respecto al grupo $A B O$, se encontró que el fenotipo más frecuente para los diferentes grupos es ccee, con un $92,3 \%$, y solo el $7,7 \%$ tienen antígenos $C$ o $E$. En el trabajo de Navarrete (8) el $92,4 \%$ de los Rh negativos el fenotipo fue ccee y sólo el 7,6\% de los donantes Rh Negativos poseen el antígeno $E$ o $C$.

Contar con donantes de sangre, voluntarios y repetitivos, fenotipados permitiría confeccionar una base de datos para proporcionar unidades de glóbulos rojos compatibles para personas ya aloinmunizadas, e idealmente prevenir la aloinmunización, especialmente en individuos que demandan transfusiones en forma crónica o en niñas y mujeres en edad fértil (2).

\section{CONCLUSIONES}

Tanto a nivel nacional como a nivel mundial el fenotipo más frecuente para las personas $R h D$ negativo es el ccee.

\section{REFERENCIAS}

1. Majluf, C. A., \& Pérez, Ó. Hematología Básica. México: GARMARTE, S.A. DE C.V, 2006.

2. Vásquez Rojas, M. Castillo Espinosa, D. Pavez Espinoza, Y. Frecuencia de antígenos del sistema sanguíneo $\mathrm{Rh}$ y del sistema Kell en donantes de sangre. Revista 
Cubana de Hematol, Inmunol y Hemoter. 2015;31(2):160-171.

3. Daniels G, Castilho L, Flegel WA, Fletcher A, Garratty G, Levene C, Et at. International Society of Blood Transfusion Committee on terminology for red blood cell suface antigens: Macao report. Vox sang 2009, 96:153-156.

4. Rivas, J y Sucre, W. Determinación de Transfusiones Sanguíneas con Fenotipos del Sistema Rh Incompatibles, Analizados Mediante Pruebas Inmunohematológicas en Receptores y Unidades De Sangre Transfundidas, remitidas por El Banco De Sangre Fausto Castello De La Cruz Roja Provincial De Napo.Quito; 2014.

5. McClelland DBL, P. E. Manual uso óptimo de componentes sanguíneos. España; 2011.

6. Alvarado V. Dubon M. Tipificación de Antígenos Eritrocitarios del Sistema Rh y Kell en Donadores de Sangre que asistieron a dos Hospitales de la Ciudad de Guatemala. Universidad de San Carlos de Guatemala. 2012.

7. Elsayid M, Al Qahtani FS, Al Qarni AM, Almajed F, Al Saqri F, Qureshi S., et al.: Determination of the frequency of the most immunogenic Rhesus antigens among Saudi donors in King Abdulaziz Medical City - Riyadh. J Nat Sci Biol Med. 2017 Jan- Jun; 8(1):56-59.

8. Navarrete, $R$ y Segura, D. Frecuencia de fenotipos del sistema rh-hr en donantes rh negativos en el Hospital SanVicente de Paul. Rev. Med. de costa rica y Centroamérica LXIX. 2012; 143-147.

9. Mantilla Gutierrez, C. Cardona Arias, J. Pérez Escobar R. Caracterización clínica y hematológica de donantes a repetición de un Banco de Sangre de MedellínColombia, 2011. Medicina y Laboratorio. Volumen 18 (9-10): 459 - 470.

10. García, M., Sáenz, E., \& Cruz, J. Estudio de factores socioculturales relacionados con la donación voluntaria de sangre en Las Américas. Revista Panamericana de Salud Pública. 2003; vol 13: 85- 90.

11. Cruz Bermúdez HF. Moreno Collazo JE. Forero SE. Caracterización de donantes voluntarios de sangre por grupo sanguíneo $A B O$ y $R h$ que asistieron a un banco de sangre de la ciudad de TunjaColombia; Volumen 12 ( $\left.\mathrm{N}^{\circ} 2\right)$ : 185 - 189.

12. Organización Mundial de la Salud. Disponibilidad y seguridad de la sangre a nivel mundial. Nota descriptiva; junio 2017. Manual de Normas Técnicas, Administrativas y de procedimiento de Banco de Sangre. Resolución 901/1996 de 20 de marzo. 